\title{
Ultraviolet femtosecond and nanosecond laser ablation of silicon: ablation efficiency and laser-induced plasma expansion
}

\author{
Xianzhong Zeng ${ }^{\mathrm{a}, \mathrm{b}}$, Xianglei Mao ${ }^{\mathrm{a}}$, Ralph Greif ${ }^{\mathrm{b}}$, Richard E. Russo*a \\ ${ }^{a}$ Lawrence Berkeley National Laboratory, Berkeley, CA, USA 94720 \\ ${ }^{b}$ Dept. of Mechanical Engineering, University of California at Berkeley, Berkeley, CA, USA 94720
}

\begin{abstract}
Femtosecond laser ablation of silicon in air was studied and compared with nanosecond laser ablation at ultraviolet wavelength $(266 \mathrm{~nm})$. Laser ablation efficiency was studied by measuring crater depth as a function of pulse number. For the same number of laser pulses, the fs-ablated crater was about two times deeper than the ns-crater. The temperature and electron number density of the pulsed laser-induced plasma were determined from spectroscopic measurements. The electron number density and temperature of fs-pulse plasmas decreased faster than ns-pulse plasmas due to different energy deposition mechanisms. Images of the laser-induced plasma were obtained with femtosecond time-resolved laser shadowgraph imaging. Plasma expansion in both the perpendicular and the lateral directions to the laser beam were compared for femtosecond and nanosecond laser ablation.
\end{abstract}

Keywords: Laser ablation, Laser-induced plasma, Shock wave, Laser-induced breakdown spectroscopy

\section{INTRODUCTION}

The interaction of a high power laser beam with material has many applications including chemical analysis, micromachining and pulse laser deposition of thin films. The laser-material interaction involves complex processes of heating, melting, vaporization, ejection of atoms, ions and molecules, shock waves, plasma initiation and plasma expansion. The resulting crater and laser-induced plasma are dependent on the laser beam parameters (pulse duration, energy, and wavelength), the solid target properties and the surrounding environment's condition ${ }^{1}$.

Lasers with femtosecond pulse duration are of particular interest for ablation as the pulse duration is less than the typical thermalization characteristic time of a few picoseconds. Due to a much smaller thermal diffusion depth, high-precision ablation and minimal damage can be obtained with femtosecond lasers ${ }^{2,3}$. Because of its very short pulse duration, the laser beam does not interact with the laser-induced plasma. A shorter plasma lifetime was reported for infrared femtosecond laser induced plasma than in the case of longer laser pulses ${ }^{4,5}$. Absorbed laser energy is fully deposited into the target and higher efficiency of material ablation can be obtained ${ }^{6}$.

The interaction of a high-energy ultraviolet (UV) pulsed laser beam with solid materials has been studied extensively at nanosecond time scales ${ }^{7-10}$. However, there only are few reports about UV femtosecond laser ablation of solids. With small absorption and thermal diffusion lengths, UV femtosecond laser pulses can minimize thermal effects and be applied to direct fabrication of microstructures ${ }^{11}$.

In this study, UV femtosecond laser ablation of silicon in air was performed and compared with UV nanosecond laser ablation. Laser ablation efficiency was compared by measuring crater depth as a function of pulse number. The laserinduced plasma properties were determined from spectroscopic measurements. Images of plasma expansion were recorded with the laser shadowgraph imaging technique.

*Correspondence: Email: rerusso@lbl.gov 


\section{EXPERIMENTAL SYSTEM}

A diagram of the spectroscopic experimental system is shown in Fig. 1. A quadrupled Nd:YAG laser (Coherent, Infinity) operating at $266 \mathrm{~nm}$ with a 3-ns pulse-width and a Ti: sapphire femtosecond laser (Spectra-Physics, TSA) with a 100 -fs pulse-width were used as the ablation lasers. The femtosecond laser beam at its fundamental wavelength ( 800 $\mathrm{nm}$ ) passed a Third Harmonic Generator (Spectra-Physics, TP-1A) to form the $266 \mathrm{~nm}$ beam that was focused on the silicon sample with a quartz lens to a spot diameter of $\sim 50 \mu \mathrm{m}$. The ambient air was at $1 \mathrm{~atm}$. pressure. The laser energy was $220 \mu \mathrm{J}$ per pulse or $\sim 11 \mathrm{~J} / \mathrm{cm}^{2}$ for both the femtosecond and nanosecond laser tests. Single pulse laser ablation was performed on silicon samples. A second lens was used to image the laser-induced plasma onto the entrance slit of a Czerny-Turner spectrometer (Spex Industries Model 270M). Spectral emission was detected by an Intensified ChargeCoupled Device (ICCD) system which consisted of a thermoelectrically cooled CCD (EG\&G Princeton Applied Research Model OMA VISION) with $512 \times 512$ pixels and a Microchannel Plate (MCP) image intensifier. This detection system provided a spectral window of $\sim 13 \mathrm{~nm}$ and resolution of typically $0.125 \mathrm{~nm}$, using an entrance slit width of 20 micron and grating with 1800 grooves $/ \mathrm{mm}$. The spatial resolution of the ICCD spectrometer plus lens arrangements was 11 microns per pixel. The dark current background of the ICCD detector was subtracted from the measured spectroscopic data for each measurement.

Gating the ICCD and changing the delay time enabled the spectra to be temporally resolved. The gate width and time delay were controlled by the OMA SPEC 4000 software (EG\&G Princeton Applied Research), and synchronously triggered from the Nd:YAG laser. The gate width was set at $30 \mathrm{~ns}$. A photo diode and a digitizing oscilloscope were used to calibrate the time delay.

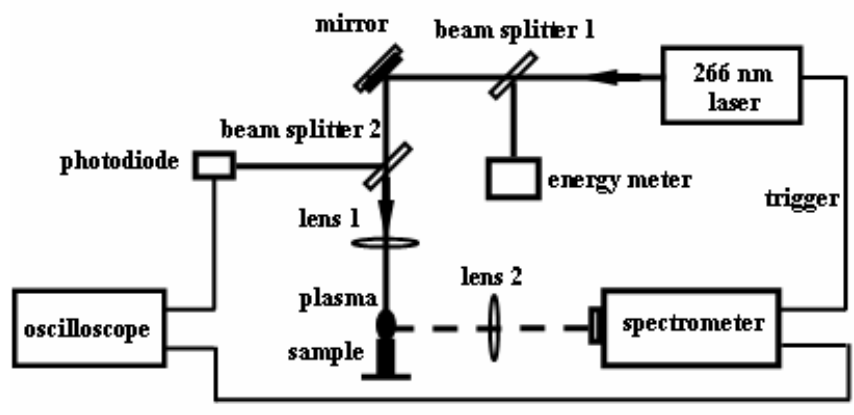

Fig. 1. Experimental system for spectroscopic measurements of laser-induced plasma.

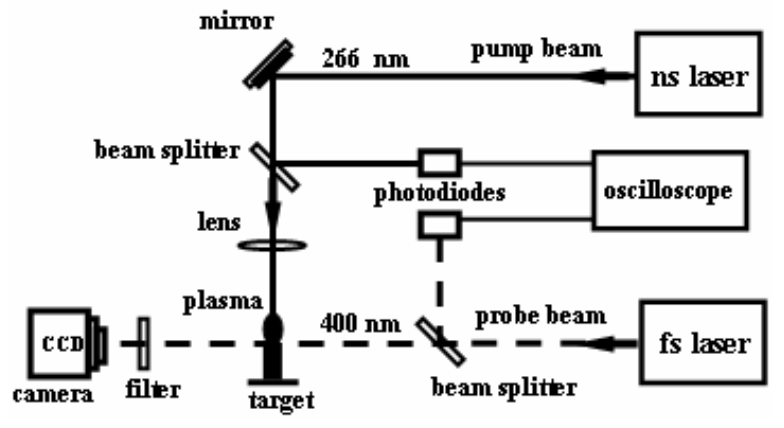

Fig. 2. Schematic of the experimental setup of the pumpprobe technique for imaging of laser-induced plasma.

The imaging experimental system is shown in Fig. 2. The nanosecond laser was used as the pump (ablation) beam and the femtosecond laser was used as the probe beam. The $800 \mathrm{~nm}$ beam of the femtosecond laser passes a potassium dihydrogen phosphate (KDP) crystal, forming a probe beam at $400 \mathrm{~nm}$ that is perpendicular to the ablation laser beam. The probe beam is directed to a CCD camera (Photometrics, AT200) after passing through a narrowband $400 \mathrm{~nm}$ filter. The time delay between the fs probe beam and ns ablation beam was controlled using a delay generator (Stanford Research, DG 535). The actual delay time was measured using photodiodes. To obtain shadowgraphs of the femtosecond laser-induced plasma, a beam splitter and an optical delay stage were used. One femtosecond laser beam $(266 \mathrm{~nm})$ was used as the pump beam, and the other one $(400 \mathrm{~nm})$ was used as the probe beam which passed through the optical delay stage before being directed to the CCD camera. By moving the delay stage, the optical path of the probe beam was varied, which changed the time delay between the pump and probe beams. The time resolution achieved with the optical delay stage can be as small as $100 \mathrm{fs}$.

\section{RESULTS AND DISCUSSION}




\subsection{Ablation depth}

Fig.3 shows the dependence of crater depth on the laser pulse number. For both fs and ns laser ablation, the crater depth increases linearly with the pulse number for this low number of pulses. For the same number of laser pulses, however, the fs-crater is about two times deeper than the ns-crater using the same fluence. The nanosecond laser generated a significant raised rim caused by resolidification of molten silicon. The rim around the fs-crater is much smaller than that of the ns-crater, indicating that melting was reduced using femtosecond pulses. Scanning Electron Microscopy (SEM) images of the laser generated particles were shown in Fig. 4. Particles from nanosecond laser ablation were mostly single large droplets ejected from the melted surface with diameters ranging from several hundreds nanometers to several microns. Primary particles generated using femtosecond laser ablation were small nano-sized spheres and agglomerates from these spheres, again suggesting that melting and ejection of molten mass is less for femtosecond laser ablation. In the femtosecond regime, the laser pulse terminates before any material escapes from the surface. The laser energy is deposited in the sample target without a laser-plasma interaction. For nanosecond laser ablation, plasma shielding results in a decrease in the laser energy reaching the surface. Thus, the ablation efficiency decreases for nanosecond laser ablation ${ }^{4}$.

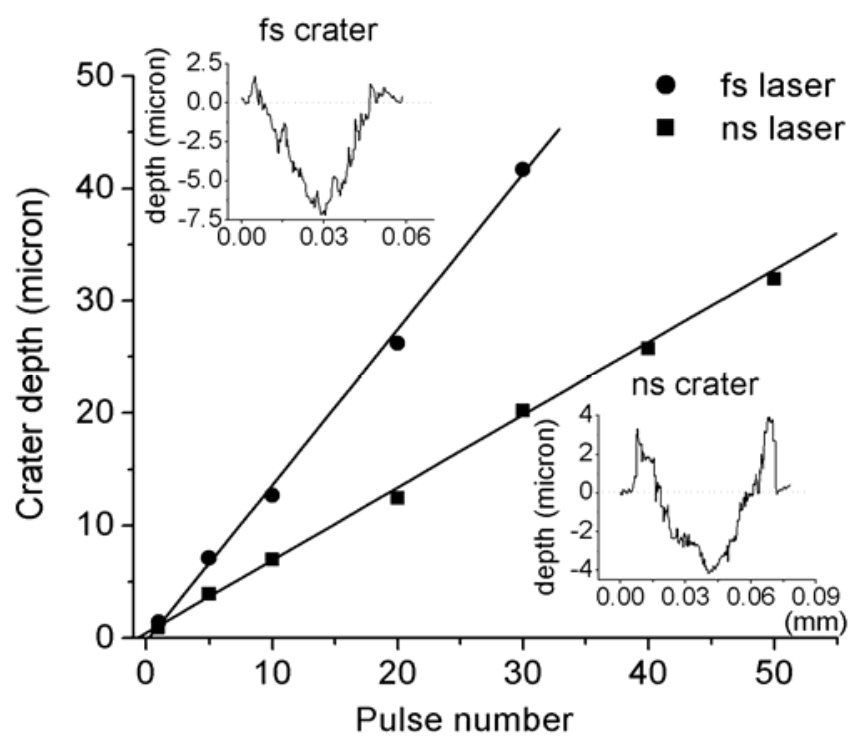

Fig. 3. Ablation depth vs. pulse number for femtosecond and nanosecond laser ablation. The insets show the fs and ns crater profile after five laser pulses.
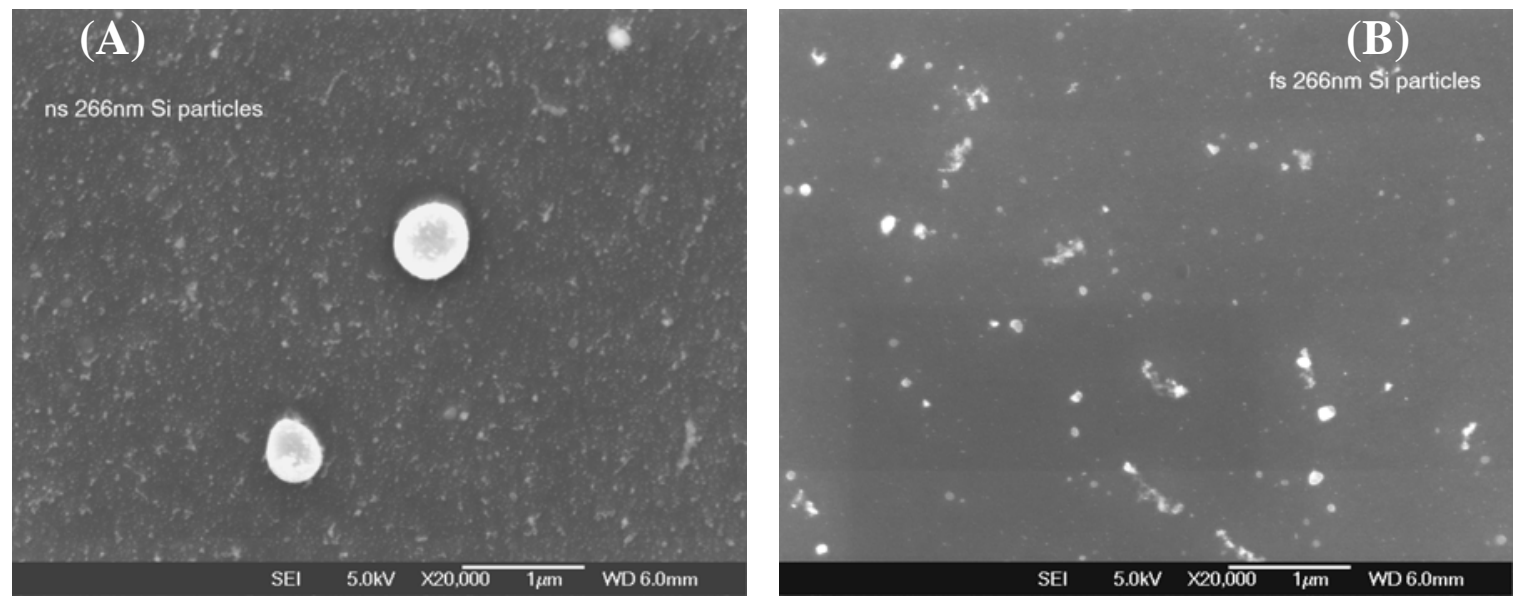

Fig. 4. SEM images of laser generated particles for (A) nanosecond and (B) femtosecond laser ablation of silicon. Particles were collected on a silicon substrate. 


\subsection{Plasma temperature and electron number density}

The silicon emission line $\mathrm{Si}(\mathrm{I})$ at $288.16 \mathrm{~nm}$ was measured for diagnosing plasma properties (plasma temperature and electron number density). Fig. 5 shows the time dependence of the Si line intensity measured with fs- and ns-pulses at a distance of $0.6 \mathrm{~mm}$ above the sample surface. After $30 \mathrm{~ns}$, the emission intensities of both fs- and ns-plasma decrease exponentially. For small time ( $<30 \mathrm{~ns})$, the emission intensity of the ns-plasma increases with time while the emission intensity of the fs-plasma decreases. At the beginning of the plasma expansion the ns-plasma becomes hotter because of absorption of the trailing part of the laser pulse via electron-neutral, electron-ion inverse bremsstrahlung and photoionization $^{12}$. For the fs-pulse, the plasma expands without any other heating process and its emission intensity decreases.

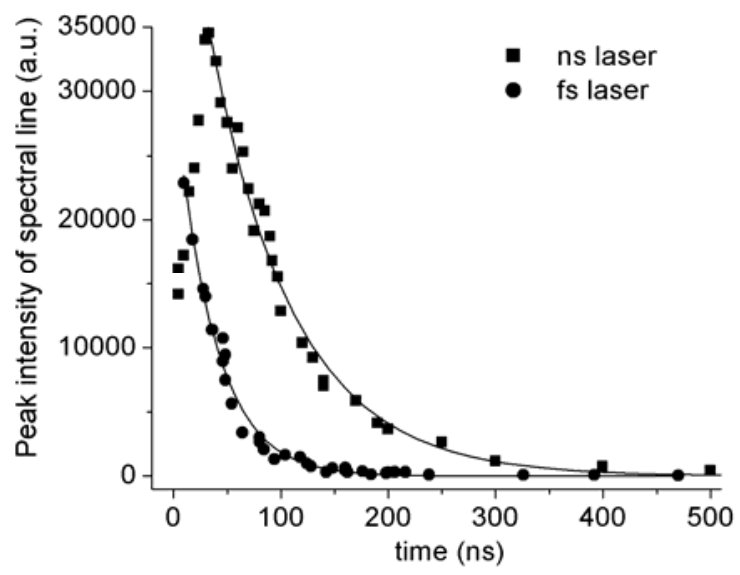

Fig. 5. Peak intensity of Si (I) $288.16 \mathrm{~nm}$ emission line as a function of time for femtosecond and nanosecond laser ablation at a distance of $0.6 \mathrm{~mm}$ above the target surface.

Assuming local thermal equilibrium (LTE), the electron temperature $T_{e}$ is assumed to be equal to the excitation temperature $T_{e x c}$. The plasma temperature $T$ can be determined by the relative line-to-continuum intensity ratio ${ }^{13}$. Stark line broadening from collisions of charged species is the primary mechanism influencing the emission spectra in these experiments $^{14,15}$. A Lorentz function was used to fit the spectra and the Full Width Half Maximum (FWHM) of Stark broadened lines and the line to continuum emission ratio were obtained and were used to determine the electron number density and temperature of the plasmas ${ }^{16}$.

The lower limit for the electron number density needed to collisionally maintain the energy-level populations to within $10 \%$ of LTE while competing with radiative decay is given by the McWhirter criterion ${ }^{17}$ :

$n_{e}\left(\mathrm{~cm}^{-3}\right) \geq 1.4 \times 10^{14}(\mathrm{kT})^{1 / 2} \Delta E^{3}$

where $T$ is the temperature and $\Delta E$ is the energy difference between the upper and lower states. For the $\mathrm{Si}(\mathrm{I})$ transition at $288.16 \mathrm{~nm}, \Delta E=4.3 \mathrm{eV}$, and at the highest temperature $k T=3.4 \mathrm{eV}$, the lower limit for $n_{e}$ is $2.1 \times 10^{16} \mathrm{~cm}^{-3}$, which is approximately two orders of magnitude lower than the value of $n_{e}$ deduced from Stark broadening. Therefore, the validity of the LTE assumption is supported. However, it should be noted that the McWhirter's criterion is a necessary but not sufficient condition for LTE.

Fig. 6 shows the temporal behavior of the electron number density and temperature of the fs-plasma and the ns-plasma. The density and temperature of the fs-plasma were less and decrease faster compared with ns-plasma due to different energy deposition mechanisms. At the femtosecond time scale, no laser-plasma interactions take place. Thus, the plasma cools quickly since no further energy is being supplied to the plasma. In contrast, a fraction of the laser energy is absorbed by the ns-plasma, which leads to greater plasma temperature and electron density. These results are consistent with ref. 6 which used femtosecond laser with $800 \mathrm{~nm}$ wavelength. 


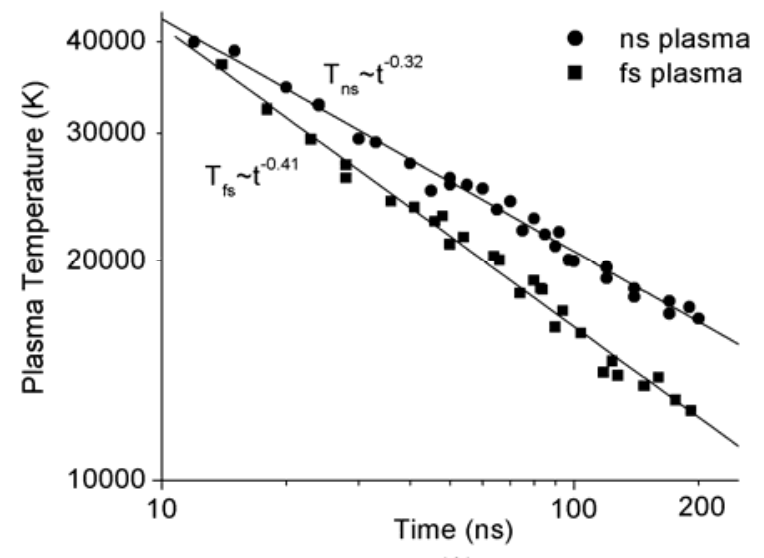

(A)

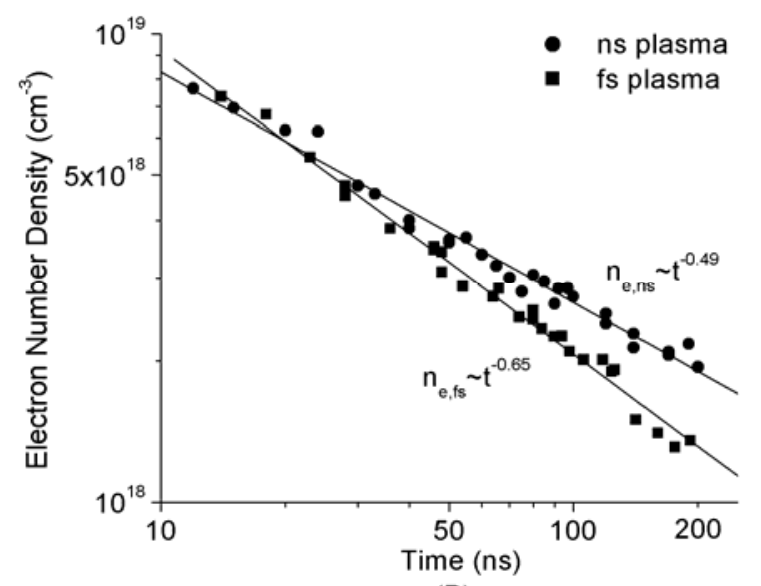

(B)

Fig. 6. Plasma temperature (A) and electron number density (B) as a function of time for femtosecond and nanosecond laser ablation at a distance of $0.6 \mathrm{~mm}$ above the target surface.

\subsection{Plasma expansion}

The propagation of the laser-generated shock wave is approximated as resulting from an instantaneous, massless point explosion (blast wave). The expansion distance $R$ between the surface of the target and the position of a blast wave depends on the energy converted into the plasma state $E$. A relation between $R$ and $E$ is obtained from the Sedov-Taylor scaling ${ }^{18,19}$ :

$R=\lambda\left(\frac{E}{\rho}\right)^{1 /(2+\beta)} t^{2 /(2+\beta)}$,

where $\lambda$ is a constant approximately equal to unity, $\rho$ is the mass density of the undisturbed air and $t$ is the time of propagation. The assumptions for the validity of this relation are that the pulse duration is shorter than $t$ and the diameter of the spot area of the laser radiation is smaller than $R$. The parameter $\beta$ represents the dimensionality of propagation (for spherical propagation $\beta=3$, for cylindrical propagation $\beta=2$ and for planar propagation $\beta=1$ ).

Fig. 7 shows shadowgraphs of the shock waves generated by femtosecond and nanosecond lasers $10 \mathrm{~ns}$ after the laser pulse. Due to edge diffraction of the probe laser beam, there are weak fringes close to the target surface. The structure of the shock waves in air is similar to that reported by Salleo, et al. ${ }^{20}$. A thin layer of shocked air separates the shock front from the ionization front. Between the ionization front and contact front is the shocked and ionized air. Laserinduced air breakdown was seen for femtosecond laser ablation due to the much larger laser irradiance of $112 \mathrm{TW} / \mathrm{cm}^{2}$, compared with the irradiance of $3.7 \mathrm{GW} / \mathrm{cm}^{2}$ for the ns laser.

Fig. 8 shows shadowgraphs of the shock waves generated by femtosecond and nanosecond lasers at different times. The spatial and temporal scales of the images are different because of the different expansion properties for fs and ns laserinduced plasmas. The shock wave generated by the nanosecond laser expands spherically (Fig. 8 e-h). Fig. 9 (A) shows that the perpendicular expansion distance of the shock wave generated by the nanosecond laser is proportional to $t^{2 / 5}$, as predicted by Sedov's blast wave theory for spherical propagation; while the perpendicular location of the shock wave generated by the femtosecond laser is proportional to $t^{2 / 3}$, corresponding to one dimensional expansion. Lateral expansion distance of the shock wave as a function of time is shown in Fig. 9 (B). At small times (less than $1 \mathrm{ns),} \mathrm{the} \mathrm{fs-}$ plasma expanded primarily in the direction perpendicular to the target surface. After several nanoseconds the fs-plasma 
expands in both the lateral and perpendicular directions. The expansion in the perpendicular direction was faster than in the lateral direction. For the ns laser, the laser-induced plasma expanded in both directions at similar velocities.
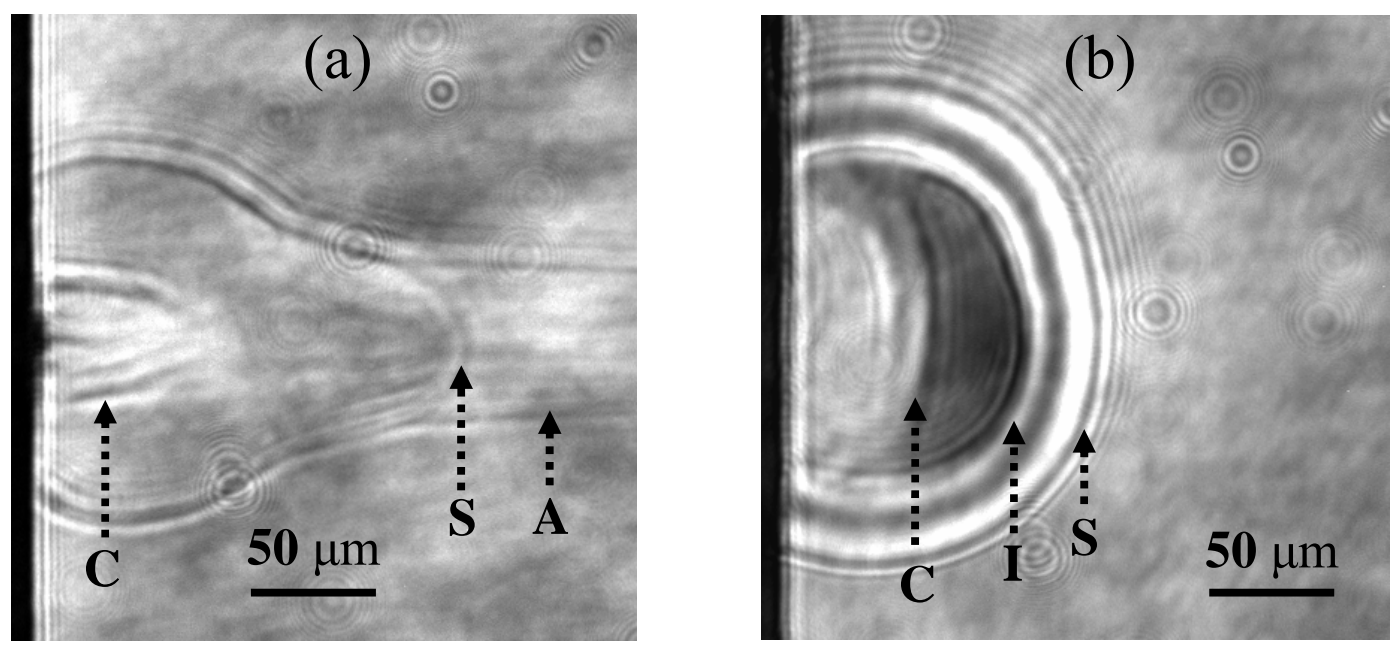

Fig. 7. Plasma images obtained by laser shadowgraphy for femtosecond (a) and nanosecond (b) laser ablation at $10 \mathrm{~ns}$ after laser shot. The shock front (S), ionization front (I), contact front (C) and air breakdown plasma (A) are labeled in the figures.

The shock wave front velocity was obtained by differentiating equation (2). At 5 ns the perpendicular expansion velocities were $13.6 \times 10^{5} \mathrm{~cm} / \mathrm{s}$ and $7.9 \times 10^{5} \mathrm{~cm} / \mathrm{s}$ for fs- and ns-plasmas, respectively. The fs-plasma kinetic energy is therefore higher than the ns-plasma at small times. According to the shock wave equations ${ }^{18,21}$, the pressure and temperature of the shocked air behind the shock front were calculated also to be higher for the fs case. As shown in Fig. 5 , the plasma temperature of fs-plasma is approximately the same with the ns-plasma temperature at $\sim 15 \mathrm{~ns}$. Since the temperature of the fs-plasma decreases faster than for the ns-plasma, the plasma temperature of fs-plasma would be higher at times earlier than $\sim 15 \mathrm{~ns}$, which is consistent with the above velocity measurement.

\section{CONCLUSIONS}

Laser ablation efficiency and laser-induced plasma properties were studied for UV femtosecond and nanosecond laser ablation of silicon. Crater depth measurements showed that ablation efficiency was enhanced for UV femtosecond laser pulses. The electron number density and temperature were obtained from spectroscopic measurements and showed that the fs-plasma decreases faster than the ns-plasma. Laser shadowgraphy measurements of plasma expansion showed that the femtosecond laser induced plasma expanded in one dimension at early times whereas the nanosecond laser induced plasma expanded in three dimensions.

\section{ACKNOWLEDGMENTS}

This research was supported by the U.S. Department of Energy, Office of Basic Energy Sciences, Chemical Sciences Division, under contract No. DE-AC03-76SF00098. 

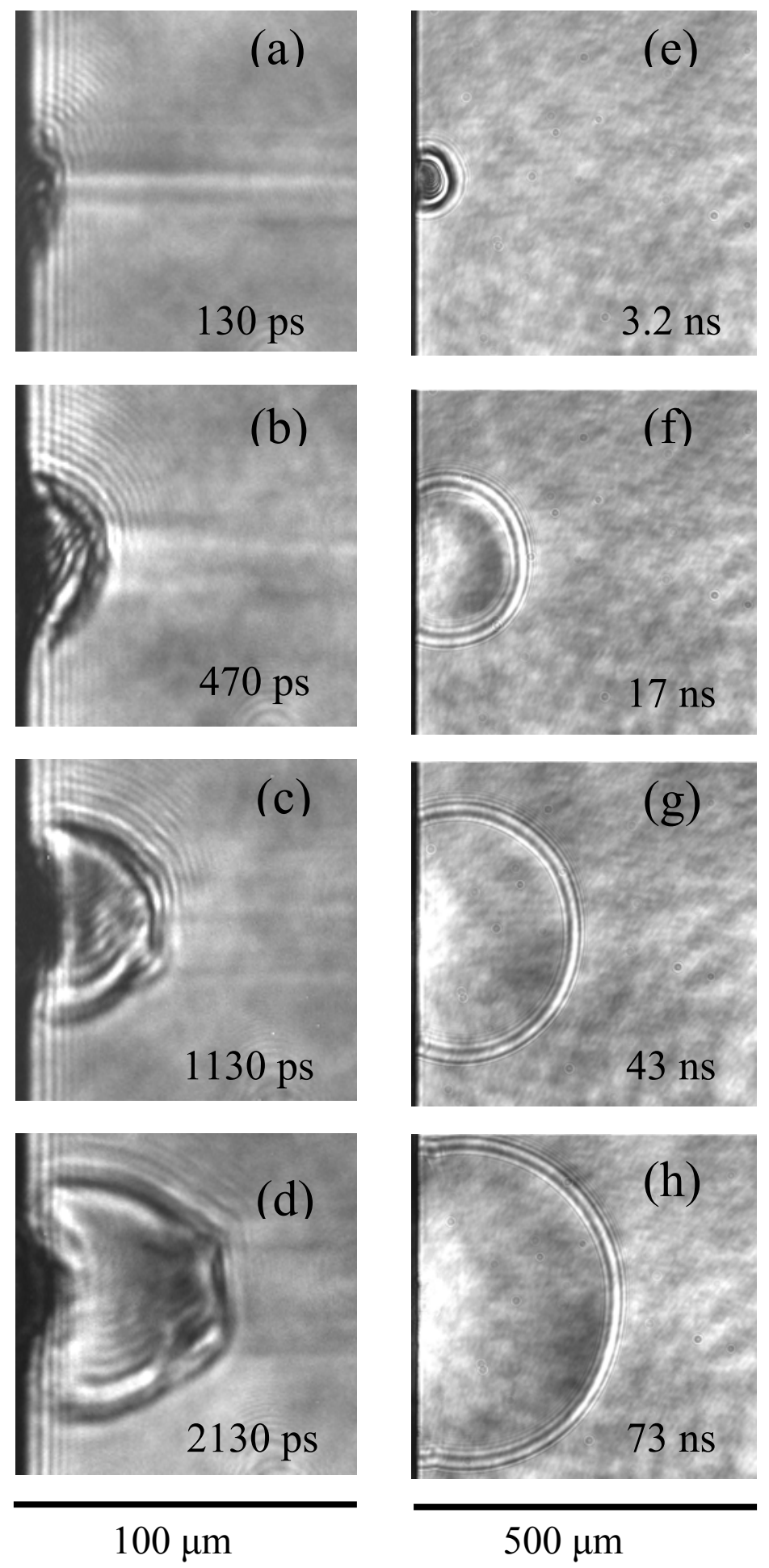

Fig. 8. Sequence of shock wave images obtained by laser shadowgraphy for femtosecond (a-d) and nanosecond (e-h) laser ablation. 


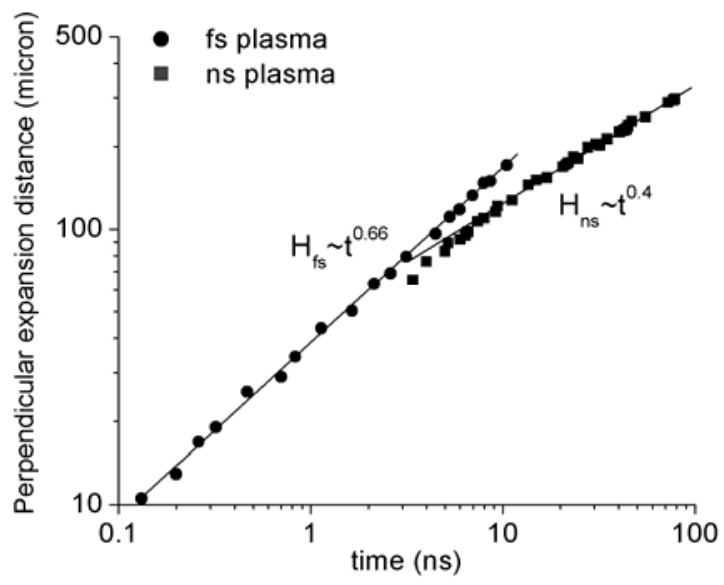

(A)

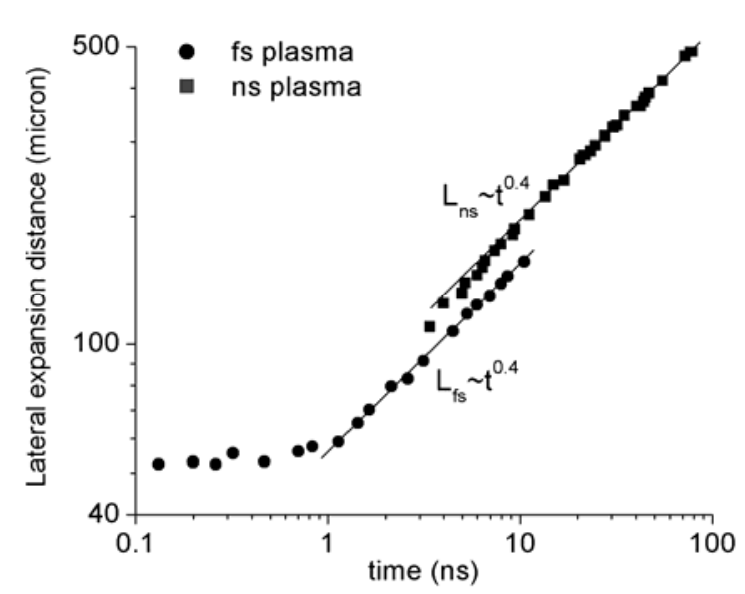

(B)

Fig. 9. Perpendicular (A) and lateral (B) expansion distance of shock wave as a function of time for femtosecond and nanosecond laser ablation.

\section{REFERENCES}

1. R.E. Russo, X. Mao, and S.S. Mao, "The physics of laser ablation in micro chemical analysis", Analytical Chemistry, 74, 70A - 77A, 2002.

2. M. Lenzner, F. Krausz, J. Kruger, W. Kautek, "Photoablation with sub-10 fs laser pulses", Applied Surface Science, 154-155, $11-16,2000$.

3. P. Rudolph, J. Bonse, J. Kruger, W. Kautek, "Femtosecond- and nanosecond-pulse laser ablation of bariumalumoborosilicate glass", Applied Physics A, 69, S763 - S766, 1999.

4. B. Salle, O. Gobert, P. Meynadier, M. Perdrix, G. Petite, A. Semerok, "Femtosecond and picosecond laser microablation: ablation efficiency and laser microplasma expansion", Applied Physics A, 69, S381 - S383, 1999.

5. V. Margetic, A. Pakulev, A. Stockhaus, M. Bolshov, K. Niemax, R. Hergenroder, "A comparison of nanosecond and femtosecond laser-induced plasma spectroscopy of brass samples”, Spectrochim. Acta Part B, 55, 1771 - 1785, 2000.

6. B. Le Drogoff, J. Margot, M. Chaker, M. Sabsabi, O. Barthelemy, T.W. Johnston, S. Laville, F. Vidal, Y. von Kaenel, "Temporal characterization of femtosecond laser pulses induced plasma for spectrochemical analysis of aluminum alloys", Spectrochim. Acta Part B, 56, 987 - 1002, 2001.

7. R.E. Russo, X.L. Mao, H.C. Liu, J.H. Yoo, S.S. Mao, "Time-resolved plasma diagnostics and mass removal during single-pulse laser ablation", Applied Physics A, 69, S887 - S894, 1999.

8. J.H. Yoo, S.H. Jeong, R. Greif, R.E. Russo, "Explosive change in crater properties during high power nanosecond laser ablation of silicon", Journal of Applied Physics, 88, 1638 - 1649, 2000.

9. S.H. Jeong, R. Greif, R.E. Russo, "Numerical modeling of pulsed laser evaporation of aluminum targets", Applied Surface Science, 129, 177 - 183, 1998.

10. H.C. Liu, X.L. Mao, J.H. Yoo, R.E. Russo, "Early phase laser induced plasma diagnostics and mass removal during single-pulse laser ablation of silicon", Spectrochim. Acta Part B, 54, 1607 - 1624, 1999.

11. I. Zergioti, S. Mailis, N.A. Vainos, A. Ikiades, C.P. Grigoropoulos, C. Fotakis, "Microprinting and microetching of diffractive structures using ultrashort laser pulses", Applied Surface Science, 138-139, 82 - 86, 1999.

12. M.V. Allmen, Laser-beam Interactions with Materials, Springer-Verlag, New York, 1987.

13. G.J. Bastiaans, R.A. Mangold, "The calculation of electron density and temperature in Ar spectroscopic plasmas from continuum and line spectra", Spectrochim.Acta Part B, 40B, 885 - 892, 1985.

14. G. Befeki, Principles of laser plasmas, Wiley Interscience, New York, 1976.

15. H.R. Griem, Spectral Line Broadening by Plasmas, Academic, New York, 1974. 
16. Xianzhong Zeng, Xianglei Mao, Samuel S. Mao, Jong H. Yoo, Ralph Greif, Richard E. Russo, "Laser-plasma interactions in fused silica cavities", Journal of Applied Physics, 95, 816 - 822, 2004.

17. R.W.P. McWhirter, in: R.H. Huddlestone and S.L. Leonard (Eds.), Plasma Diagnostic Techniques, Academic, New York, 1965.

18. L.I. Sedov, Similarity and Dimensional Methods in Mechanics, Cleaver Hume, London, 1959.

19. Gert Callies, Peter Berger, Helmut Hugel, "Time-resolved observation of gas-dynamic discontinuities arising during excimer laser ablation and their interpretation”, J. Phys. D: Appl. Phys., 28, 794 - 806, 1995.

20. A. Salleo, F.Y. Genin, M.D. Feit, A.M. Rubenchik, T. Sands, S.S. Mao, R.E. Russo, "Energy deposition at front and rear surfaces during picosecond laser interaction with fused silica", Applied Physics Letters, 78, 2840 - 2842, 2001.

21. Xianzhong Zeng, Xianglei Mao, Sy-Bor Wen, Ralph Greif, Richard E. Russo, "Energy deposition and shock wave propagation during pulsed laser ablation in fused silica cavities", Journal of Physics D: Applied Physics, 37, 1132 1136, 2004. 